\section{Roles de género: incidencia en las decisiones de los y las estudiantes ${ }^{1}$}

\author{
Andrea María Alas Hernández ${ }^{2}$ \\ Silvia Maricela Ramos Ramos ${ }^{3}$
}

\author{
Palabras clave: \\ roles de género, especializaciones \\ técnicas, modelaje externo, rol productivo \\ y reproductivo, identidad de género.
}

\begin{abstract}
Resumen
Esta investigación intentó comprender el fenómeno de la persistencia de los estudiantes hombres y mujeres por elegir especializaciones técnicas relacionadas con los roles de género, ya que, según datos de los últimos cinco años, los hombres optan, en su mayoría, por carreras como mecánica o electricidad, mientras que las mujeres eligen enfermería o servicio al cliente. El estudio se enfocó en los jóvenes que estudian especializaciones en Educación Media Técnica en dos instituciones públicas de San Salvador y San Luis Talpa, La Paz. Uno de los principales hallazgos fue que los aprendizajes de género construidos en la familia influyen en la elección de la especialización, pero no determinan la decisión.
\end{abstract}

\section{La persistencia de elegir especializaciones por género}

La investigación sobre la igualdad de género en el área educativa se ha fortalecido en los últimos años y, en El Salvador, el tema es de suma importancia tanto para el ámbito educativo como en el contexto social del país porque persisten las desigualdades de oportunidades entre mujeres y hombres. De igual forma, la temática de la investigación enfocada en los roles de género aprendidos por los estudiantes tiene relevancia, ya que, en el país, se acentúa el comportamiento reproductivo para las mujeres para generar roles de sumisión y dependencia, mientras que en los

$1 \quad$ Alas H., A. M. y Ramos R., S. M. (2021). Relación entre la identidad de género construida en la familia y la elección de la especialización técnica [Tesis de Maestría]. Universidad Centroamericana José Simeón Cañas.

2 Maestra en Política y Evaluación Educativa.

3 Maestra en Política y Evaluación Educativa. 
hombres es el comportamiento productivo el que genera autoridad e independencia.

Ejemplo de ello es que, según datos proporcionados por la Encuesta de Hogares de Propósitos Múltiples de 2017, las mujeres en El Salvador tienen una participación laboral del $46 \%$, mientras que la de los hombres es del 80 \% (DYGESTIC, 2017); es decir que la participación de los hombres en el sector laboral, y productivo, es casi el doble que el de las mujeres. Estos datos también tienen una coincidencia con la elección de especialización técnica en bachillerato (o Educación Media), pues existe una amplia brecha entre las mujeres que eligen especializaciones técnicas frente a los hombres.

Por ejemplo, entre el 2015 y el 2018, la especialidad de Atención Primaria de Salud fue elegida por más mujeres: el $75 \%$ de los estudiantes eran mujeres y el $25 \%$ eran hombres. Esta área implica el cuidado de pacientes enfermos y atención en casos que requieren primeros auxilios. Por otra parte, la especialización en Desarrollo de Software, la cual implica el aprendizaje de conocimientos y habilidades en el campo tecnológico, ha sido elegida por más hombres. Entre el 2015 y el 2018, casi el $70 \%$ de los estudiantes matriculados fueron hombres, y solo el $30 \%$ mujeres.

Con estos datos, se permite profundizar en que las especializaciones altamente técnicas son elegidas especialmente por hombres, mientras que las que implican cuidado y atención son elegidas en su mayoría por las mujeres. Por tanto, se presenta un fenómeno donde los roles productivos y reproductivos que se identifican a nivel social también se reproducen en el plano educativo.

Sin embargo, cabe mencionar que, en el ámbito educativo, existen esfuerzos por disminuir las brechas de desigualdad de género. Uno de estos es la Política de Equidad e Igualdad de Género del Ministerio de Educación de El Salvador (MINED, 2020), la cual es la única política nacional que busca disminuir la elección de especializaciones por roles de género.
Sin embargo, existen vacíos en dicha política, en los cuales la investigación trató de profundizar. El principal vacío fue conocer la vinculación que existe entre la identidad individual de género y las expectativas sociales construidas en la familia con la elección de especializaciones de Educación Media Técnica de estudiantes de dos institutos públicos de San Salvador y La Paz.

Con esto, se trató de profundizar en la vinculación de los roles de género aprendidos en la familia sobre la elección de especialización técnica, ya que consideramos que la política no profundiza sobre las relaciones familiares de los estudiantes que realizan las elecciones y, como ya se mencionó antes, los roles de género, productivos y reproductivos, tienen su origen en las relaciones sociales.

También existe un vacío sobre la obtención de datos directos de estudiantes de Educación Media Técnica, ya que este grupo de informantes no había sido investigado previamente en la política educativa de género en el país.

Por lo tanto, algunos de los hallazgos de esta investigación y que podrán ayudar a enriquecer la Política de Equidad e Igualdad de Género son que la elección de especialización es un proceso complejo influenciado por diversos factores, tales como: a) los roles de los padres y las madres, b) la construcción de la identidad de género, c) las expectativas de género de los padres, d) los modelajes externos de profesionales y e) el apoyo de la madre. Con lo anterior, se evidencia que la decisión de elegir una especialización vinculada o no al género no se toma con unos días de antelación a la matrícula en las instituciones educativas, sino es un proceso que se construye durante un periodo previo.

Por otra parte, esta investigación también tiene una vinculación con las políticas de equidad $e$ igualdad de género que se implementan a escala centroamericana, ya que uno de los hallazgos permite reflexionar sobre la importancia de que las niñas desde temprana edad se involucren en áreas como la ciencia, la tecnología, la ingeniería, las matemáticas, 
tanto para su desarrollo personal como para el desarrollo de sus comunidades.

Esto se enlaza con el Plan de Acción para la Igualdad entre los Géneros de UNICEF (2018), el cual se encuentra vigente actualmente y uno de sus principales desafíos es impulsar la enseñanza secundaria de las niñas, dando prioridad al desarrollo de competencias en las áreas mencionadas.

De la misma forma, el estudio se vincula con los objetivos 4 y 5 de los Objetivos de Desarrollo Sostenible de Naciones Unidas. Lo anterior se debe a que estos buscan promover la igualdad de oportunidades entre los géneros y empoderar a las mujeres y a las niñas (CEPAL, 2019). En esta investigación, se demostró que las niñas consultadas han crecido con inseguridades en sus capacidades para desarrollarse en áreas de ciencia y tecnología, por lo que se concluye que es fundamental formarlas en sus seguridades y en el empoderamiento, sobre todo en estas áreas de estudio.

Por otra parte, es importante destacar que la investigación también se relaciona con otros escritos a escala regional de países como México y Colombia, que han buscado fortalecer el vínculo entre las políticas educativas y la igualdad de género. En ambos países, algunas universidades han desarrollado investigaciones para conocer las razones por las cuales mujeres y hombres eligen especializaciones basadas en sus roles de género, $y$ con los resultados se busca aportar resultados que tengan incidencia en la construcción de la política educativa local.

\section{Marco conceptual}

Danermark et al. (2016) manifiestan que "una parte esencial del trabajo de las ciencias sociales consiste en cómo analizar, desarrollar el lenguaje teórico y utilizarlo en el análisis empírico" (p. 206), ya que, según el realismo crítico, el marco teórico apoya la interpretación de los resultados de la investigación. Tomando en cuenta esta premisa, se presentó, de forma breve, los roles de género en la familia, la construcción de la identidad individual de género en el contexto familiar y las expectativas sociales de género desde una mirada internacional. Además, se mostró un marco contextual en El Salvador del objeto de estudio, haciendo énfasis en la Educación Media Técnica.

El contexto local nos indicó que los roles de género en la familia del país tienen ciertas cualidades y se manifiestan a través de diferentes acciones. Una de esas principales particularidades es la división entre la asignación social de los roles, reproductivos para las mujeres y productivos para los hombres. Esto quiere decir que, en las familias, la mujer cumple con el rol de procrear hijos y educarlos, además de ser servicial y dar cuidado. Esto ha perdurado durante los años. Muestra de ello es la investigación realizada por Ignacio Martín-Baró (1980), en la que demuestra que los roles estaban arraigados en las ideas de los estudiantes universitarios; en sus resultados, el autor explica: "[A] la hora de señalar la virtud más importante en el hogar, se tiende a esperar del hombre la responsabilidad global, mientras que en la mujer se enfatiza relativamente más su capacidad para dar amor y para cumplir con su rol materno" (p. 562).

El MINED ha identificado estos roles de género. Esto se evidenció en la Política de Equidad e Igualdad de Género, pues uno de sus ejes de acción está enfocado en la disminución de la división sexual en la Educación Media Técnica, ya que muestran un problema enfocado en la desigualdad de mujeres y hombres inscritos en las diferentes opciones: "Continúa predominando una división sexual de los estudios, especialmente en educación media, además de una insuficiente incorporación y aplicación de los principios de igualdad y no discriminación en los instrumentos curriculares" (MINED, 2020, p. 36).

Según el MINED, para 2019, en Educación Media Técnica se contaba con las 41 especialidades que se encuentran activas actualmente. De todas esas especializaciones 
técnicas, para esta investigación, se han elegido únicamente dos: a) Especialización en Industria e Innovación con Mantenimiento Eléctrico y b) Especialización en Salud

Se analizó que, en la actualidad, la Educación Media tiene la visión de formar capacidades para que los estudiantes tengan más oportunidades de insertarse en el mundo laboral con mayor rapidez, tal como se presenta a continuación:

La Educación Media Técnica, partiendo de una revisión y actualización de su currículo, de la formación y capacitación de los docentes, tiene la intencionalidad de prepararse frente al ordenamiento de la economía y el proceso de modernización de la sociedad actual que genera fuertes exigencias en la formación y cualificación de recursos humanos (MINED, 2020, p. 33).

En ese sentido, en este estudio se presentó la evolución que ha tenido la Educación Media Técnica en el país y la relación con los esfuerzos por disminuir los índices de discriminación de género y la creación de especializaciones técnicas. Esto es importante, ya que en este nivel educativo hay especializaciones técnicas que, al ser seleccionadas por los estudiantes, reflejan que sí influyen los roles de género aprendidos en su familia y la identidad de género que han construido.

La relevancia de comprender el género dentro de las dinámicas sociales, especialmente dentro de estudiantes hombres y mujeres de Educación Media Técnica y su familia, es lo que brindó un marco de reflexión para analizar con criticidad aquellas relaciones sociales y culturales que se generan entre grupos de socialización y que anteriormente no se cuestionaban porque estaban normalizadas. De tal forma, Macionis et al. (2011) determinan, desde un punto sociológico, que el género no solo se refiere "a los aspectos sociales adscritos a las diferencias sexuales, sino que también implica un sentido de jerarquía y poder por parte de los hombres en cuanto a recursos sociales" (pp. 334-335). Por lo tanto, es esencial comprender cómo y cuándo estas diferencias son asignadas a los hombres y a las mujeres, ya que estas han generado una disparidad en las funciones que cada uno cumple en la sociedad. Hoy día, a pesar de que el rol de la mujer es más significativo, la mayoría de puestos de poder están bajo el mando de hombres; por ejemplo, en direcciones de centros educativos y ministerios del Gobierno, entre otros.

Los roles de género se han definido en el campo académico y teórico para evitar ambigüedades al momento de utilizarlos en investigación. De las diferentes definiciones que se han generado, se retoma la planteada por Galet Macedo y Alzás García (2014, citados por Almagro Navarrete, 2017), en la cual los roles de género se refieren a la forma de ser y comportarse dentro de la sociedad con base en lo que se entiende por masculinidad y femineidad. Por consiguiente, existen diferencias marcadas entre ambos géneros, para los cuales ya existen características y papeles previamente establecidos y aprendidos en el contexto en el que se desarrollan los individuos.

Lo anterior coincide con el estudio de enseñanza y psicología que presentan Aguilar Montes de Oca et al. (2013), en el cual los roles de género se explican como "la forma en la que se comportan y realizan su vida cotidiana hombres y mujeres, según lo que se considera apropiado para cada uno" (p. 209). Las definiciones de roles de género que se han presentado en este estudio tienen similitudes en cuanto al comportamiento, la división entre hombre y mujer, y la pertinencia y la relevancia del contexto.

Para profundizar, se revisaron teorías sobre el aprendizaje de los roles de género, es decir, teorías que buscan explicar desde la psicología y la sociología cómo se aprenden los roles: a) teorías cognitivas, con enfoque psicológico, y b) teorías del aprendizaje social, con un enfoque social. Ambas explican perspectivas distintas acerca de la adquisición de los roles de género. 
La primera teoría acerca de la adquisición de los roles de género es la teoría cognitiva, la cual ha sido enfocada en el aspecto psicológico de los individuos. Uno de los principales promotores de las teorías cognitivas fue Kohlberg (1981). Estas teorías proponen que "las diferencias de género surgen por un proceso de categorización por el que los niños y las niñas se sitúan a sí mismos en la categoría masculina y femenina ordenando sus experiencias" (Macionis et al., 2011, p. 340). Lo anterior surge de manera casi inconsciente. Otro aspecto importante que plantean estas teorías es que, a través de estas experiencias, los niños y las niñas van construyendo sus identidades individuales de género; esto es significativo, ya que dependiendo de cada una de estas experiencias los roles de género son dinámicos.

La segunda teoría es conocida como la teoría del aprendizaje social. Esta se considera como la más apropiada para este estudio, debido a sus características y semejanzas con el objeto de estudio planteado. El principal representante de esta teoría es Albert Bandura. El investigador generó una serie de características adjudicadas a esta teoría. La primera característica revela la importancia del entorno social en el aprendizaje de los roles de género; la segunda es la adquisición de conocimiento, valores, normas, cultura, actitudes y destrezas a través de la observación. Esta característica da pauta para que la tercera se cumpla, ya que esta describe cómo el niño y la niña son capaces de aprender observando a través del modelaje. La última característica relevante es la interacción existente entre los individuos y el contexto en el que aprenden; se dice que la conducta humana es, en su mayor parte, aprendida y no innata.

Según Freixas Farré (2012), estas teorías sobre el aprendizaje social suscitan que los roles de género se aprenden por la observación y el modelaje. De acuerdo con Macionis et al. (2011), los roles de género en los niños y las niñas se definen debido al sistema de aprendizaje que se implementa; dependiendo de las actividades que hagan, así recibirán consecuencias de los demás: "[E]s un sistema que se basa en el condicionamiento operante y el aprendizaje por observación" (Freixas Farré, 2012, p. 157). Al parecer, esta teoría consiste en que los niños y las niñas reproducen los comportamientos que se observan en el ambiente en el que se encuentran y continúan comportándose según las cualidades de su género para recibir un estímulo posterior. Además, aprenden que no reproducir los comportamientos asociados a su género también generará consecuencias. A manera de conclusión, esta teoría está basada en dos grandes supuestos: a) el aprendizaje se desarrolla en el área cognitiva de los individuos, a través de asociaciones mentales y elementos simbólicos, y b) la manera de comportarse de los niños y las niñas es básicamente aprendida y no se nace con ella, sino depende del entorno, de los sujetos que los rodean y de la observación.

Considerando la teoría del aprendizaje social, los roles de género se caracterizan por ser un proceso de aprendizaje e interiorización social diferenciado para los niños y las niñas. Como en todo proceso de socialización, según Galet Macedo y Alzás García (2014), se pueden distinguir las siguientes fases: a) aprendizaje, en el cual predomina la observación y reproducción de los roles representados por parte de otros sujetos; b) interiorización, en la cual los niños y las niñas comienzan a adoptar sus roles con base en su masculinidad y feminidad; y c) transmisión, en la que aplican en la sociedad sus funciones en cuanto a género.

En este proceso intervienen diversos agentes sociales como la familia, el grupo de iguales, la escuela y los medios de comunicación. Sin embargo, en esta investigación, se considera que "la familia es la primera unidad social donde el niño se desarrolla y donde tienen lugar las primeras experiencias sociales" (Rivas, 2016, citado por Almagro Navarrete, 2017, p. 36). Por lo tanto, es primordial comprender qué se establece por familia. Para este propósito, De la Calle y Méndez (2018) proporcionan la siguiente definición: 
"La familia es un entorno social, con una serie de relaciones entre los miembros; así sean emparentados, de linaje, etc. (...) a través de las cuales se va generando un conocimiento sobre el mundo y la realidad de las relaciones; donde los vínculos afectivos son un aspecto fundamental" (p. 159).

Lo anterior sirve como base para comprender lo que Rivas (2016, citado por Almagro Navarrete, 2017) expone cuando describe que la familia es uno de los primeros agentes sociales donde el niño y la niña se desarrollan y donde tienen lugar las primeras experiencias sociales. Es decir, la familia es parte fundamental para que, desde que nacen, tanto niñas como niños aprendan cómo relacionarse en un mundo desconocido para ellos, y sus modelos a seguir no solo son sus padres, sino cada miembro de la familia al cual están expuestos. Del mismo modo, la familia coadyuva a las niñas y los niños a que adquieran más conocimiento como parte de su aprendizaje social.

\section{¿Cómo se obtuvo la información?}

Luego de la definición del fenómeno como una problemática para la política educativa, se orientó la investigación hacia el estudio de las causas que originan dicho fenómeno y se identificó que la familia es un actor clave en la formación de los estudiantes. Por lo tanto, se enfocó en conocer la construcción de roles de género en la familia nuclear y cómo se relaciona con las elecciones de especialización.

Concretamente, la inquietud que orientó la investigación fue conocer la relación que existe entre la identidad individual de género y las expectativas sociales construidas en la familia, con la elección de especializaciones técnicas.

Los informantes de la investigación fueron un grupo de ocho estudiantes de Educación Media Técnica de San Salvador y de San Luis Talpa, La Paz. Cuatro de la especialización técnica de Desarrollo e Innovación y cuatro de Enfermería. El segundo grupo de informantes fueron sus padres y madres $\mathrm{y}$, como grupo de contraste, también participaron cuatro docentes de ambas especializaciones.

Las razones por las que se seleccionó a los estudiantes son porque se buscó una fuente de información directa sobre los actores del fenómeno de estudios. Además, resultó importante analizar este grupo porque en el país no había una investigación relacionada con los roles de género que tuviera como fuente a estudiantes en este nivel educativo. La mayoría de estudios se había desarrollado con estudiantes de educación superior.

En relación con los padres de familia, estos fueron una fuente de información porque han sido los principales actores de la construcción de roles de género en las familias. Además, informaron sobre las expectativas de género sobre sus hijos, una de las categorías de análisis de la investigación.

Por su parte, los docentes brindaron información como grupo de contraste, es decir, para tener un contrapunto sobre los datos obtenidos de las primeras dos fuentes y poder brindar un segundo nivel de análisis.

Cabe mencionar que, a nivel metodológico, se utilizó el diseño intensivo (Danermark et al., 2016), el cual tiene como enfoque indagar en los mecanismos que generan el fenómeno, conocidos como "mecanismos generativos", para luego brindar explicaciones que muestren la relación existente entre dichos mecanismos.

Las principales técnicas de investigación aplicadas fueron la entrevista a profundidad y el cuestionario de diversidad. La entrevista permitió profundizar en los informantes, sus experiencias, recuerdos y percepciones personales. También fue una técnica pertinente para indagar en la decisión que realizan los estudiantes de Educación Media Técnica cuando eligen su especialización técnica relacionada con roles de género.

Además, la entrevista a profundidad se utilizó para comprender la relación entre la identidad individual de género y las expec- 
tativas sociales construidas en la familia, con la elección de especializaciones de Educación Media Técnica de estudiantes. En ese sentido, Fàbregues Feijóo et al. (2016) explican que la entrevista en profundidad consiste en un intercambio oral entre dos o más personas con el propósito de alcanzar una mayor comprensión del objeto de estudio, desde la perspectiva de los informantes, quienes en este estudio fueron los estudiantes de Educación Media Técnica, docentes en servicio y padres de familia, los cuales se perfilan en la siguiente sección.

El instrumento que se diseñó para la entrevista fue una guía de entrevista semiestructurada. Las preguntas de dicho cuestionario se realizaron en orden secuencial con los temas y fueron flexibles, pues las investigadoras tuvieron la oportunidad de repreguntar si lo consideraron necesario.

La segunda técnica que se utilizó fue la encuesta de diversidad, y se eligió con el fin de obtener diferentes perspectivas en cuanto a la información que podían proporcionar los participantes. Jansen (2013) explica que "la encuesta cualitativa es el estudio de la diversidad (no de la distribución) en una población" (p. 48). Por lo tanto, la selección de esta técnica no fue con el objetivo de promediar $\mathrm{u}$ obtener datos estadísticos.

El uso de encuestas de diversidad en metodología cualitativa, como la que se usó en la investigación, permite que este instrumento sea menos estructurado y utilizado para obtener información detallada sobre las opiniones y los razonamientos que no se identifican a simple vista en las personas en cuanto al objeto de estudio. En consecuencia, se trabajó con la encuesta de diversidad con categorías preestructuradas, las cuales permitieron propiciar el análisis de la información pertinente con el objeto de estudio.

El instrumento que se diseñó para la encuesta de diversidad fue el cuestionario preestructurado, pues por su flexibilidad permitió establecer preguntas abiertas o cerradas según las necesidades del estudio.
A pesar de que en la tradición investigativa contemporánea el cuestionario se ha caracterizado por ser un instrumento de los métodos cuantitativos, diferentes autores sugieren que se puede adaptar a las necesidades del diseño y ser completamente funcional para una investigación con cualidades cualitativas (Rodrigues Bresque et al., 2011). Con este instrumento, se buscó sondear ideas generales sobre las dimensiones del objeto de estudio, específicamente en las expectativas sociales y la elección de especializaciones técnicas de Bachillerato Técnico Vocacional en Industria e Innovación con Opción en Mantenimiento Eléctrico y Bachillerato Técnico en Salud Opción Enfermería.

El cuestionario se diseñó para ser implementado a través de una plataforma en línea; esto se debió a la flexibilidad y la sencillez que esta modalidad ofrece. Lo anterior se consideró, ya que, durante el desarrollo del estudio, los estudiantes no se encontraban recibiendo clases presenciales debido a la pandemia por la covid-19, por lo cual esta modalidad permitió que respondieran al cuestionario en dispositivos electrónicos que estaban a su alcance. Este instrumento se diseñó y difundió a través de la plataforma SurveyMonkey y los informantes lo completaron sin la observación de las investigadoras.

La aplicación de los instrumentos se realizó en un periodo de cuatro semanas a través de dos plataformas virtuales: Google Meet, que es una función de Google para realizar videollamadas que se pueden grabar, y SurveyMonkey, que consiste en una plataforma para realizar encuestas en línea.

Por otra parte, acorde al diseño intensivo de la investigación, para seleccionar y perfilar a dichos informantes, se utilizó una muestra cualitativa realista (Danemark et al., 2016; Parra, 2019). Esta muestra cualitativa realista se enfoca en "cómo, a través de ella, se demuestra la interpretación y explicación del objeto de estudio" con base en las preguntas de investigación (Emmel, 2013, citado por Parra, 2019). Es decir, una de las cualidades más importantes de la muestra cualitativa 
realista es que se perfila con base en los criterios de selección que surgen a partir de la teoría planteada sobre el fenómeno de estudio. Por lo tanto, la muestra estuvo sustentada en cualidades que provienen de la teoría. La información que se obtuvo de los informantes es compleja, pero más pertinente para la investigación, porque la muestra estuvo directamente relacionada con la teoría y las dimensiones del estudio.

En otras palabras, esta estrategia permitió a los investigadores establecer un perfil predeterminado o criterios que los participantes deben reunir con base en las premisas teóricas que se establecieron.

Tabla 1. Criterios demográficos de los participantes

\begin{tabular}{|l|l|l|}
\hline Informantes & Cantidad & Ubicación \\
\hline $\begin{array}{l}\text { Estudiantes de Educación } \\
\text { Media Técnica }\end{array}$ & $\begin{array}{l}4 \text { mujeres, } \\
4 \text { hombres }\end{array}$ & 4 del instituto público de San Salvador \\
\cline { 3 - 3 } & & 4 del instituto público de San Luis Talpa, La Paz \\
\hline $\begin{array}{l}\text { Docentes de Educación } \\
\text { Media Técnica }\end{array}$ & $\begin{array}{l}2 \text { mujeres, } \\
2 \text { hombres }\end{array}$ & 2 del instituto público de San Salvador \\
\cline { 2 - 3 } & 2 del instituto público de San Luis Talpa, La Paz \\
\hline Padres de familia & 2 madres, & 2 del instituto público de San Salvador \\
\cline { 2 - 3 } & 2 padres & 2 del instituto público de San Luis Talpa, La Paz \\
\hline
\end{tabular}

Nota: el cuadro contiene los criterios demográficos que se determinaron para elegir a los participantes. Fuente: elaboración propia.

\section{Presentación de los resultados}

Al procesar la información recolectada de los informantes, los resultados de la investigación se dividieron en dos partes: la primera fue la presentación de los resultados, donde se expuso la información respondiendo a las preguntas de investigación, pero sin interpretarla. En la segunda parte, se generó la discusión sobre los principales hallazgos.

En este apartado, se exponen los resultados de la investigación que se organizaron de acuerdo con las preguntas de investigación. En relación con la primera pregunta, "¿Cuáles son los roles de género aprendidos en la familia que definen la identidad individual de género?", se presentó la información en dos categorías: a) roles de género aprendidos en la familia, y b) definición de la identidad de género a partir de los roles.

Roles de género aprendidos en la familia

Se logró identificar que los estudiantes aprendieron los roles a través de los padres y las madres de familia. El $47 \%$ de los participantes aprendieron roles de género masculinos, mientras que el $53 \%$, roles de género femenino. Estos datos confirman el aprendizaje de roles diferenciados en la familia y ayudan a comprender que, para que se denominen roles de género, estos deben realizarse como hábitos o situaciones diarias que se reproducen ya sea por hombres o mujeres, según lo que se considera apropiado para cada uno (Aguilar Montes de Oca et al., 2013, p. 209). 
De igual forma, se identificó que el $47 \%$ de los participantes que aprendieron roles masculinos estaban relacionados con los roles productivos, mientras que el $53 \%$ de los participantes aprendieron roles reproductivos, que están relacionados con labores domésticas socialmente atribuidas a las mujeres, como limpiar y cocinar.

La identidad de género vinculada con los roles de género
En este apartado se describe cómo los roles de género que se aprenden en la familia definen la identidad de género de los y las estudiantes de Educación Media Técnica. Rocha Sánchez (2009) plantea que la identidad ha sido comprendida como atributos, rasgos o cualidades con las que se define una persona a partir de su sexo. A continuación, se presenta la información recopilada en cuanto a la identidad de género.

Figura 1. Identidad de género

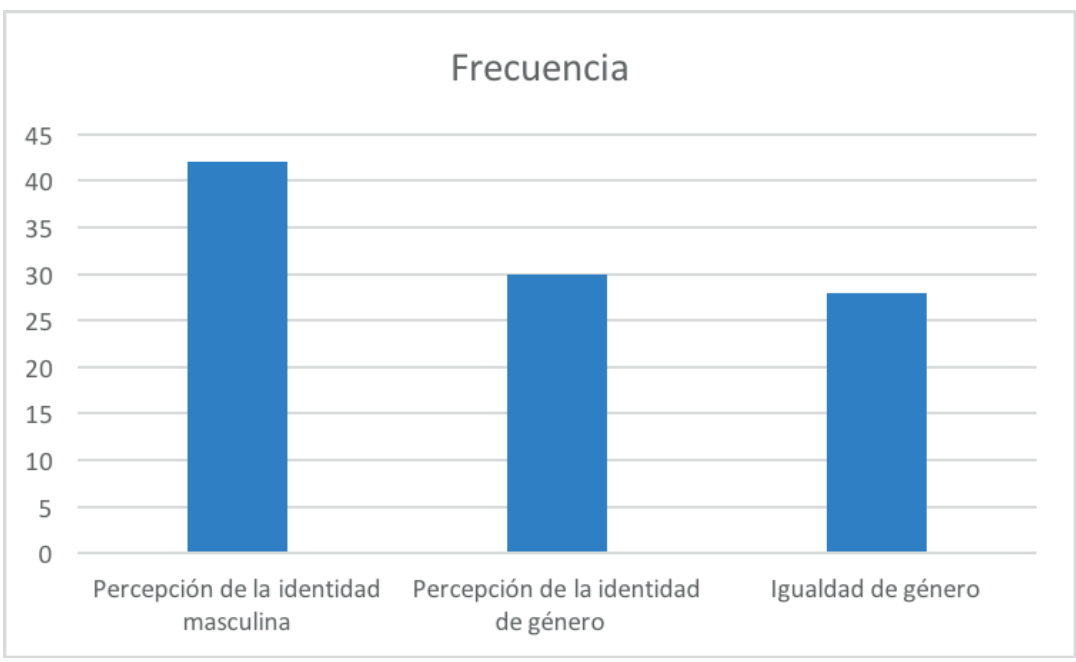

Nota: la gráfica muestra la percepción que hubo con respecto a las identidades de género masculina y femenina, asî como a la igualdad de género. Fuente: elaboración propia.

En la gráfica anterior, se ilustran tres categorías significativas para poder evidenciar cómo se relacionaron los roles de género con la identidad de género. Estas son: a) percepción de la identidad masculina, b) percepción de la identidad de género. De igual forma, se expone una categoría nueva que surgió en el procesamiento de la información: c) igualdad de género.

\section{Percepción de la identidad femenina}

La percepción de la identidad femenina que mostraron los participantes de esta investigación evidenció cómo a las mujeres se les continúa atribuyendo roles de género reproductivos y que estos constituyen la identidad de género que ellas han construido. Esto es coherente con lo definido por Chaves Jiménez (2012), cuando expresa que las mujeres aprenden una identidad de género asociada a la feminidad que las pone en desventaja, como la cualidad de hogareñas, o dependientes, porque provoca que no asuman roles de liderazgo y participación. El mismo autor expone que "ser mujer es ser dependiente, es decir, se sienten, se piensan y se representan en relación con las demás personas; y no en relación a sí mismas. Se les asignan las tareas de nutrir, comprender, proteger y sostener a otros" (Chaves Jiménez, 2012, p. 8). Ejemplo de estas percepciones femeninas son la que se presenta a continuación: 
Estudiante mujer: "Las mujeres solo tenemos que lavar los trastes, hacer limpieza, cocinar, cosa que los hombres no pueden hacer o no les gusta hacer por lo mismo que se les ha inculcado tiempo atrás y no pueden ser femeninos" (Entrevista, 2020).

\section{Percepción de la identidad masculina}

El estudiantado hizo evidente sus percepciones acerca de la masculinidad. Un aspecto importante es que los estudiantes mencionaron que, cuando llegan de trabajar, los papás deben descansar, contrario a lo que hacen las madres, ya que ellos tienen un trabajo formal y remunerado, y no están o no se ven obligados a realizar actividades del hogar como las que usualmente realizan las mujeres. Una de las opiniones de los participantes fue:

Estudiante hombre: "Pues he aprendido que cocinar no es un oficio de hombre porque tradicionalmente lo hacen las mujeres de la familia" (Entrevista, 2020).

En relación con la segunda pregunta, "¿Cuáles son las expectativas sociales de género de las familias y del estudiantado que influyen en la elección de especializaciones de Educación Media Técnica?", estas se dividen en las categorías expectativas sociales de género de los padres y las madres sobre sus hijos e hijas, expectativas que influyeron en la elección de Especialización Técnica y expectativas de género tradicionales.

Expectativas de los padres y las madres

Se identificó que hubo expectativas sociales que los padres y las madres tienen para cada género en dos áreas específicas: a) especializaciones que estudiarán y b) las áreas laborales en que se desempeñarán. Algunos ejemplos de las expectativas sociales de género que tuvieron los papás sobre sus hijos hombres están vinculadas con los roles de género que tradicionalmente se les han asignado a los hombres y a las mujeres, como se muestra en las opiniones siguientes:

Papá de estudiante mujer: "Cuando yo estudiaba bachillerato, se decía que la mujer Secretariado por ser hembra, pero no podía estudiar Contaduría, Ingeniería, que Ingeniería estudiaba el hombre" (Entrevista, 2020).

Papá de una estudiante: "Yo le dije a los profesores que cuál era la garantía o el beneficio que estaban dando con esta carrera (Industria e Innovación) porque, como dicen, verdad, esa carrera es para hombres" (Entrevista, 2020).

Expectativas que influyeron en la elección de Especialización Técnica

Por otra parte, en cuanto a las expectativas que influyeron en la elección de Especialización Técnica, los y las estudiantes manifestaron que han aprendido las expectativas de género de sus padres, es decir, expectativas tradicionales y vinculadas a sus roles de género. Las estudiantes mujeres tuvieron expectativas de que se desarrollaran en carreras como Enfermería o que estuvieran relacionadas con atención al cliente, mientras que los hombres asumieron expectativas relacionadas con sus roles de género productivos. Por ejemplo, en las especializaciones que implican fuerza física, la mayoría de estudiantes opinó que son para hombres: 
Figura 2. Resultados especializaciones que implican fuerza

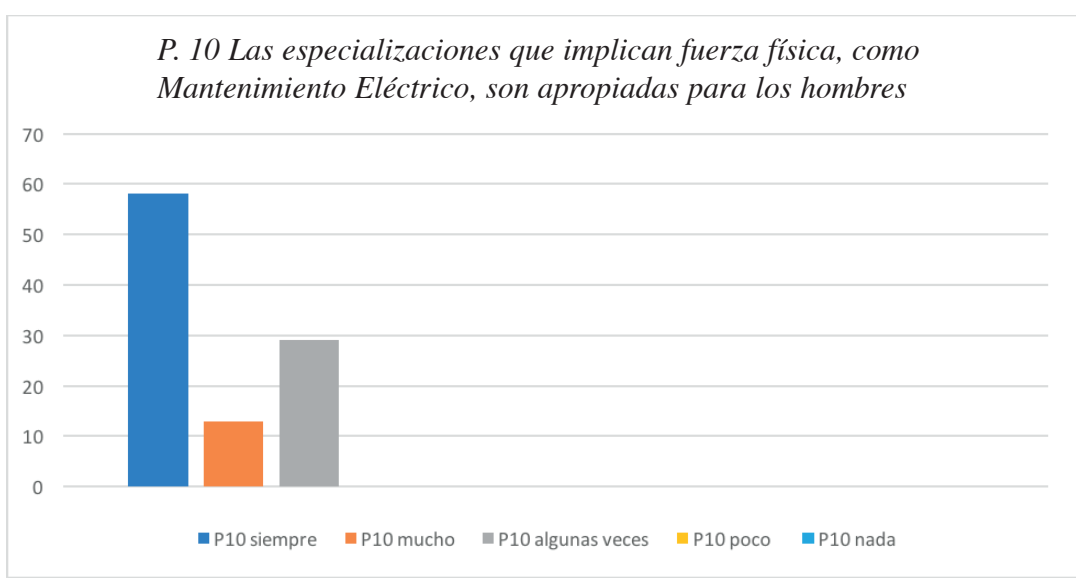

Nota: la gráfica presenta los resultados referentes a especializaciones que implican fuerza. Fuente: elaboración propia.

Casi el 60 \% señaló la respuesta "siempre" y más del 11 \% marco "mucho", aduciendo que los hombres pueden optar por este tipo de especializaciones. Por otra parte, existen especializaciones que los y las participantes consideraron que son apropiadas para las mujeres.

Figura 3. Resultados de la especialización de Enfermería para mujeres

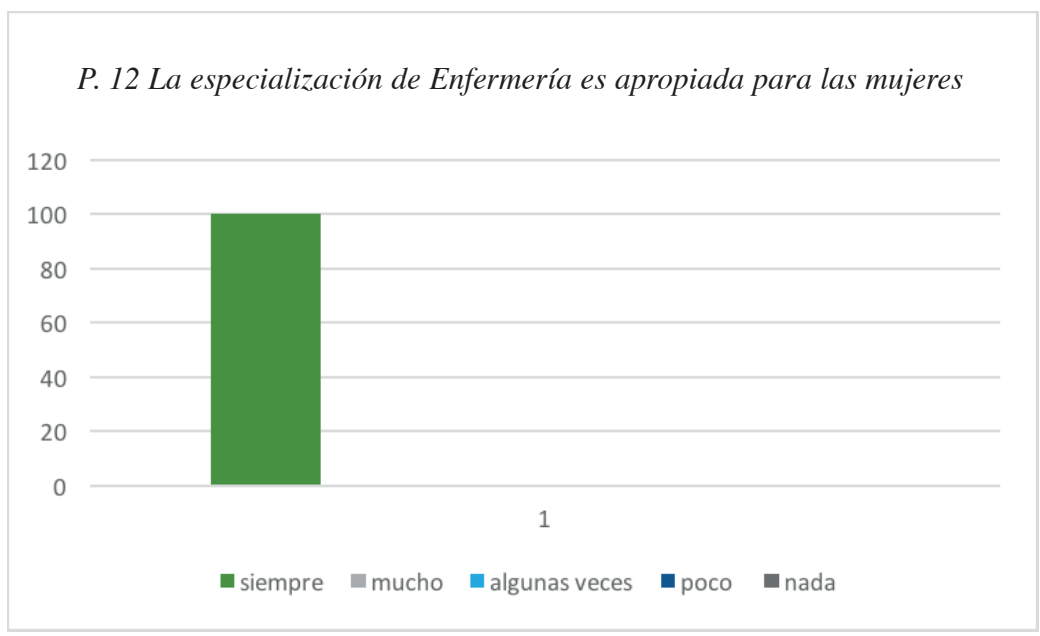

Nota: la gráfica expone los resultados ofrecidos con respecto a si la especialización en Enfermería es apropiada para mujeres. Fuente: elaboración propia.

Todos los estudiantes consideraron que la especialización de Enfermería "siempre" es apropiada para las mujeres; esto coincide con las representaciones sociales de que en
Enfermería solo laboran mujeres. Del mismo modo, con respecto a la misma pregunta, pero consultada en relación con los hombres, se percibieron diferencias. 
Figura 4. Resultados especialización Enfermería para hombres

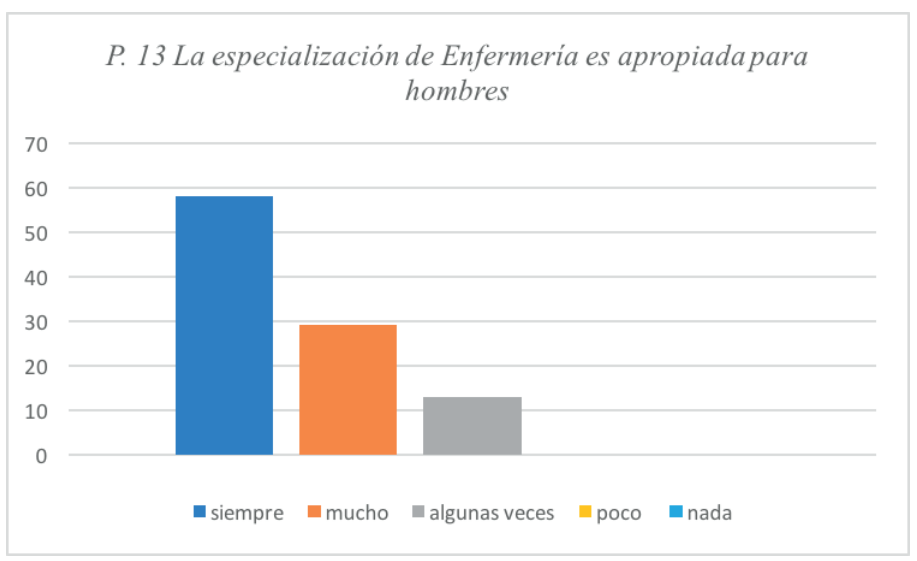

Los hombres consideraron que Enfermería puede ser apropiada "siempre" en casi un $60 \%$, pero también algunos opinaron que esta puede ser apropiada sólo "algunas veces" en un porcentaje superior al $10 \%$. Este es un dato interesante porque permite conocer otra perspectiva, y es que más de la mitad de los hombres consultados sí consideran que Enfermería es apropiada para ellos. Además, cuando se les cuestionó sobre Mantenimiento Eléctrico, y de si para las mujeres era una especialización más complicada, contestaron de la forma siguiente:

Figura 5. Resultados especialización Mantenimiento Eléctrico para mujeres

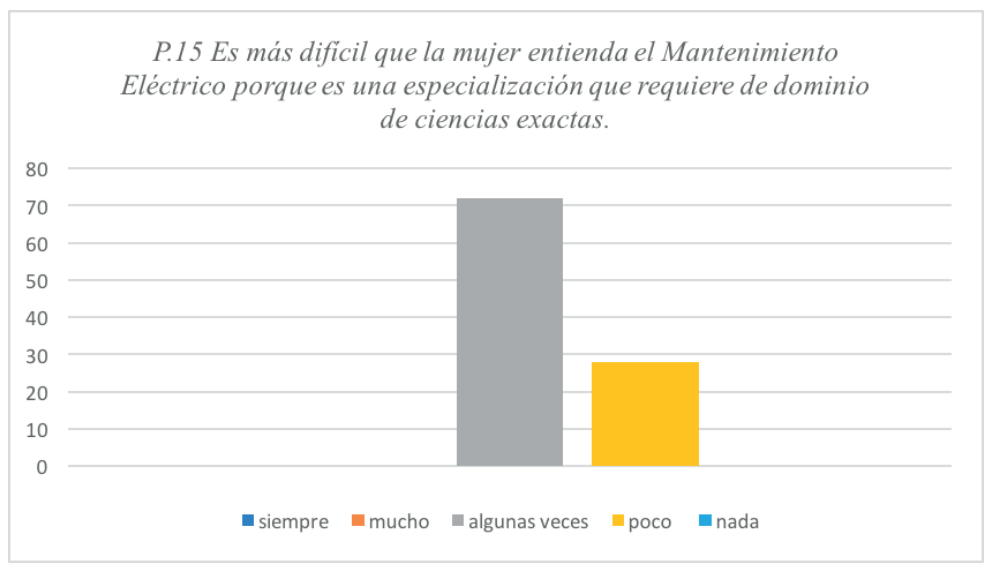

Nota: la gráfica muestra los resultados sobre el entendimiento de las mujeres con respecto a la especialización en Mantenimiento Eléctrico. Fuente: elaboración propia.

El $72 \%$ de los estudiantes consideran que, para la mujer, "algunas veces" es difícil que entienda el Mantenimiento Eléctrico, mientras que el $28 \%$ dijo que es "poco" difícil que lo entienda. Ello deja en evidencia que no se cuenta con seguridad por parte de los participantes de si las mujeres requieren dominio de ciencias exactas. 


\section{Los roles de género influyen, pero no determinan}

La tesis principal de este artículo está estrechamente vinculada con el principal hallazgo de la investigación. La tesis que se plantea es que, si bien los estudiantes tienen un aprendizaje de roles de género con sus familias, se evidenció que dicho aprendizaje sí tiene una influencia sobre las decisiones de los estudiantes, como comportamientos sociales y perspectivas; sin embargo, este aprendizaje de roles de género, y la influencia de género de sus papás, no determina la elección de una especialización técnica. Esto debido a que existen influencias externas, como el modelaje externo, el discurso emergente sobre igualdad de género y el apoyo de la madre que genera casos disruptivos.

Esta afirmación es importante, ya que la literatura previa indicaba que el aprendizaje de roles de género en las familias es una construcción sólida y arraigada en los comportamientos, decisiones e ideas de las personas (Almagro Navarrete, 2017). De igual forma, constituye un condicionante para las decisiones que se toman en el futuro.

Asimismo, bajo un razonamiento de lógica inmediata, se intuye que el aprendizaje de género en las familias crea un perfil de persona que se mantendrá constante en el tiempo y se manifestará principalmente en los comportamientos del individuo (Galet Macedo y Alzás García, 2014). Sin embargo, con los hallazgos, se determina que no siempre es así, ya que este aprendizaje es influyente, pero no determinante.

A la vez, eso resulta valioso porque abre una oportunidad de intervención para la política educativa, pues es una oportunidad donde se puede actuar para que cambie la situación de la incidencia de elección de especialización por roles de género.

Para profundizar en esta afirmación, cabe destacar que los estudiantes tienen un aprendizaje de roles de género en sus hogares. Desde que son niños, aprenden acciones concretas para hombres y mujeres, especialmente en los oficios, pues las mujeres se encargan de cocinar, lavar y limpiar; mientras que los hombres consideran idóneas las acciones que implican fuerza física, como actividades de construcción, fontanería o electricidad.

Estas ideas y acciones se van apropiando con más fuerza a través del tiempo. Es decir que, a mayor edad, el individuo tiene más arraigadas las ideas de roles de género. Dicha afirmación se vincula con la teoría de la identidad individual de género, según la cual la construcción de la identidad personal permite que se vayan interiorizando y apropiando los roles de género (Galet Macedo y Alzás García, 2014).

Por lo tanto, los jóvenes informantes ya tenían cierto nivel de arraigo con los roles de género aprendidos en sus familias nucleares. Se identificó que ellos tenían un sentido de pertenencia con cualidades socialmente atribuidas a las mujeres y a los hombres. Ejemplo de lo anterior es que, en el caso de las mujeres, estas indicaron que tenían rasgos más sentimentales y que eran más ordenadas $y$ atentas, porque en sus hogares, desde pequeñas, aprendieron a limpiar, ordenar y servir a su familia. Mientras que los hombres explicaron que se sentían identificados con cualidades masculinas, las cuales describieron como ser fuertes físicamente, tener valentía, liderar y tener independencia económica. Un ejemplo de esto es la opinión siguiente:

Estudiante hombre: "Ser femenino es para las mujeres; como hay igualdad, ellas pueden trabajar, pero no deben hacer cosas de mucha fuerza y deben encargarse del hogar, ser delicadas" (Entrevista, 2020).

De igual forma, el aprendizaje de roles de género va más allá de las acciones y los comportamientos que ejecutan, pues también influye a nivel cognitivo; es decir, en las decisiones, percepciones y perspectivas que los jóvenes tienen sobre sus vidas y las de los demás (Sánchez, 2009). 
Ejemplo de ello es la perspectiva de género que tienen sobre las profesiones, ya que consideraban que las carreras profesionales de cuidado, atención y servicio eran idóneas para las mujeres, pero las especialidades que implicaban fuerza física, liderazgo o procesos cognitivos relacionados con las matemáticas eran preferibles para los hombres. Lo anterior se muestra en las opiniones a continuación:

Papá de estudiante mujer: "Cuando yo estudiaba bachillerato, se decía que la mujer Secretariado por ser hembra, pero no podía estudiar Contaduría, Ingeniería, que Ingeniería estudiaba el hombre" (Entrevista, 2020).

Papá de una estudiante mujer: "Yo le dije a los profesores que cuál era la garantía o el beneficio que estaban dando con esta carrera (Industria e Innovación) porque, como dicen, verdad, esa carrera es para hombres" (Entrevista, 2020).

Esto se vincula directamente con las ideas que sus papás tenían sobre sobre las profesiones. Todos los papás hombres consultados consideraron que las mujeres debían seleccionar carreras que no implicaran fuerza física, riesgos o procesos cognitivos complejos, ya que ellas tenían capacidades pertinentes con especializaciones donde pudieran atender o cuidar a los demás.

Para los hombres, sí consideraban que debían estudiar carreras altamente técnicas, con las que pudieran aspirar a un empleo que les diera roles de liderazgo, o que les permitiera un crecimiento económico, como especializaciones altamente técnicas. Por lo tanto, es debido a estas razones que se considera que los estudiantes consultados tuvieron un sólido aprendizaje en roles de género con sus familias, y en esta investigación se logró constatar que los aprendizajes influyen en la construcción de su identidad de género, ya que ellos se han apropiado de los atributos de lo masculino y lo femenino.
Por ende, al contar con un sólido aprendizaje en roles de género, se considera que este influye en las decisiones, perspectivas $y$ acciones que los jóvenes realizan. Sin embargo, en una decisión tan importante como la elección de especialización técnica, este aprendizaje sí influye, pero no lo determina; es decir, no marca la causa de la decisión final. Por lo tanto, resulta válido hacer la pregunta: ¿qué otros elementos están influyendo en los estudiantes para que tomen la decisión de elegir una especialización asociada, o no, a los roles de género?

Se identificó que la elección de especialización técnica también está influenciada por modelajes externos, específicamente modelajes profesionales de lo que los estudiantes aspiran a ser como profesionales en el futuro. Los hombres estudiantes de Enfermería explicaron que ellos, cuando eran niños, tuvieron experiencias en los hospitales, donde fueron atendidos por médicos hombres. Con el tiempo, dichas experiencias se convirtieron en aspiraciones profesionales de lo que ellos deseaban estudiar.

De igual forma, las mujeres estudiantes de Enfermería, cuando eran niñas, fueron atendidas por mujeres enfermeras, quienes fueron la inspiración que tuvieron para estudiar dicha profesión. Sin embargo, el modelaje externo que tuvieron se establece sobre los roles de género, ya que las mujeres tuvieron una influencia de modelaje de las enfermeras, mientras que los hombres, de los doctores. Esto se refleja en los aportes de los participantes que se muestran a continuación:

Estudiante hombre: "En mi mente decía yo quiero ser como el doctor, poder hacer todo lo que él hace por sus pacientes" (Entrevista, 2020).

Estudiante mujer: "Cuando yo estaba bien pequeña me enfermaba bastante seguido, entonces siempre me fijaba cómo las enfermeras tomaban la temperatura, les hacían un 
diagnóstico a sus pacientes, eso me atrajo" (Entrevista, 2020).

Por ello, a pesar de que hombres y mujeres optaron por la especialidad de Enfermería, en realidad aspiraban a tener profesiones diferentes: las mujeres querían ser enfermeras, una profesión más apegada a los cuidados y la atención; mientras que los hombres aspiraban a ser doctores, una carrera más enfocada en el liderazgo, y la capacidad de dirigir diferentes procedimientos médicos.

Continuando con otra de las razones externas que influyeron en la elección de especialización técnica, es importante mencionar que se identificó que existe un discurso emergente sobre igualdad de género en los jóvenes consultados.

Este suceso es significativo porque, como bien se menciona anteriormente, los jóvenes han pasado por un sólido aprendizaje de roles de género en sus hogares; por lo tanto, a pesar de esto, ellos tienen un discurso de igualdad. Este se percibe cuando ellos hablan, o cuando se les consultó sobre temas vinculados a la equidad de género. Según indicaron, debería existir igualdad de oportunidades entre hombres y mujeres, o están de acuerdo en que no deberían existir restricciones solo por ser hombre o ser mujer.

Los jóvenes cuando hablaron sobre esto se sentían seguros y daban la impresión de que realmente estaban apropiados de sus opiniones y valoraciones. Sin embargo, cuando se les consultó sobre sus acciones vinculadas a la equidad de género, se notó una diferencia, porque ahí se demostró que en sus acciones no estaba presente la equidad de género que manifestaban en su discurso.

Un ejemplo sencillo de estas acciones es que, en la práctica, siguen haciendo actividades domésticas socialmente asignadas a hombres y mujeres, o no conviven mucho tiempo con el sexo opuesto porque consideran que tienen cualidades diferentes. Por lo tanto, luego de un análisis, se descubrió que este fenómeno no solo se ha registrado en El Salvador, sino también en otros países como México, donde han identificado que los estudiantes tienen un discurso sobre igualdad de género, pero no lo demuestran en sus prácticas.

Es por ello por lo que se designó que los estudiantes tienen un discurso emergente de género (y únicamente un discurso), ya que las acciones no son coherentes con la igualdad. Con esto no se quiere decir que, en un futuro, el discurso pueda transcender a las acciones concretas. Esto también demuestra que los procesos de aprendizaje son dinámicos y cambiantes, ejemplo es el surgimiento de un discurso más equitativo aun en un contexto de aprendizaje de roles y expectativas de género.

Finalmente, el elemento que también permite explicar las razones de no elegir especializaciones basadas en los roles de género está vinculado con casos especiales, o también llamados casos disruptivos. Estos se refieren a estudiantes que, a pesar de tener influencia de los roles de género, eligieron especializaciones opuestas a las que tradicionalmente se espera de un hombre o una mujer.

Estos casos disruptivos se refieren, concretamente, a las mujeres que eligieron Mantenimiento Eléctrico, y a los hombres que seleccionaron Enfermería. Desde la elección de especialización, demostraron que algo había pasado durante su aprendizaje de roles de género para que optaran por una carrera profesional diferente.

Pero al indagar más en las razones, se descubrió que, efectivamente, tuvieron un aprendizaje diferente en su hogar. Las niñas fueron formadas sobre procesos técnicos como electricidad y matemática. Esto influyó en la elección de una carrera altamente técnica.

Los estudiantes hombres de Enfermería tuvieron experiencias de atención hacia familiares o vecinos y crecieron con un apego mayor a sus mamás. Cuando eligieron una especialización técnica, este acercamiento influyó en que seleccionaran un área no vinculada con roles de género masculinos. 
Estos casos son particularmente interesantes porque las decisiones de la mamá son opacadas por las del papá, es decir que, en los casos analizados, las decisiones importantes las toma el papá y la madre solo las acepta. Sin embargo, cuando el hijo tiene un mayor acercamiento con la mamá y ella puede acompañarlo en las decisiones, los estudiantes sienten mayor seguridad de tomar las decisiones con las que sí se sienten cómodos y poder superar el condicionamiento de elegir una especialización que esté vinculada a su género.

Estos casos se documentaron concretamente en los estudiantes hombres de Enfermería, quienes coincidieron que tenían un mayor apego a su madre y eso les dio más seguridad para elegir una carrera técnica diferente.

Por lo tanto, los casos disruptivos tienen a la base un elemento de formación, y es que los niños recibieron formación alternativa a la que tradicionalmente se le enseña a un hombre y una mujer.

Lo valioso y significativo de estos casos disruptivos es que rompen con el esquema tradicional de reproducción y aprendizaje de roles tradicionales de género. Lo anterior permite conocer áreas donde puede intervenir la política educativa para que el fenómeno de la elección influida por roles tradicionales de género comience a presentar cambios y se tengan oportunidades más equitativas para estudiantes mujeres y hombres a través de mediaciones oportunas.

Finalmente, un elemento que también influye en la elección de especialización técnica es la expectativa de género que tienen los padres y las madres sobre los hijos hombres y mujeres, el cual está íntimamente relacionado con lo que se espera de ellos en términos laborales.

El papá hombre espera que sus hijos hombres estudien una especialización vinculada a los roles de género, es decir que implique fuerza física o que sea altamente técnica. Para sus hijas mujeres, tienen la expectativa de que se formen en las humanidades o en carreras donde puedan cuidar a otras personas, como Enfermería, y no esperan que ellas se encuentren en carreras que les pueda generar mayor remuneración económica, ya que su destino no será liderar equipos, o incluso en su propia familia no se encargará de tener el rol productivo. Esto demuestra que aún existe un rechazo a la idea de que la mujer tenga mayor representación y participación en dos áreas que tradicionalmente han estado dominadas por los hombres: el saber y el poder.

El saber, en el sentido del acceso a conocimientos que pueden abrir mejores oportunidades de desarrollo profesional y personal, y el poder, en la forma en que las mujeres pueden influir en las decisiones que tienen impactos a nivel social. Esto es pertinente destacarlo porque, en el país, existen datos que demuestran que las mujeres tienen baja representación en puestos de poder como en alcaldías (aun cuando se exige una cuota de género) o en la Asamblea Legislativa.

Dichas expectativas están relacionadas íntimamente con el ámbito laboral y, nuevamente, con los roles productivos y reproductivos. Del hombre, sus padres esperan que estudie especializaciones altamente técnicas porque esto les permitirá tener más ingresos para que puedan ser jefes de hogar y ejercer el rol productivo. Por su parte, las mujeres pueden optar por carreras menor remuneradas, ya que ellas no tienen la obligación de proporcionar el mayor ingreso económico a su familia y, en su lugar, sí deben tener tiempo para atender las labores domésticas de su casa y cuidar a los miembros de la familia, es decir, continuar desarrollando el rol reproductivo.

Sin embargo, los padres están condicionando a sus hijos a que cumplan con una estructura de género que presenta desigualdades, discriminaciones, diferencias e inequidades, donde especialmente las mujeres han sido afectadas al ser relegadas a cumplir acciones específicas y no tienen libertad de hacer funciones o actividades de forma plena. 
Esto coincide con el discurso restrictivo que tienen los docentes hombres sobre las mujeres estudiantes de Mantenimiento Eléctrico, pues, según la información que brindaron, mencionaron que las mujeres no tendrán las mismas oportunidades que los hombres en esa carrera, ya que implicaba el uso de la fuerza física, y ellas no tenían esa cualidad. De igual forma, destacaron que ellas debían limitarse a estudiar carreras donde pudieran servir a los demás.

En su discurso, se identificó un elemento restrictivo para las mujeres, pues constantemente mencionaron las acciones que ellas no debían hacer; y para los hombres, el discurso era diferente porque para ellos sí consideraban que había más oportunidades de elegir especializaciones, como el área tecnológica. Por su parte, las docentes mujeres sí manifestaron un mayor apoyo hacia las estudiantes mujeres, e indicaron que eran capaces de desempeñarse en dicha área.

Esta situación refleja que tanto los padres como los docentes hombres consultados en esta investigación tienen expectativas de género que limitan a las mujeres, ya que consideran que solo pueden desempeñarse en las áreas consideradas como femeninas. Para los estudiantes hombres, hay más libertades, pero de igual forma sigue siendo un discurso restrictivo para ellos porque no les permite desarrollarse en las áreas que ellos prefieren, sino solo en las que se consideran como masculinas.

Por lo tanto, dicho discurso afecta a los y las estudiantes, permea en sus propias expectativas y se convierte en condicionantes que influyen al momento de elegir una especialización técnica. Aunque ellos deseen estudiar algo alejado a sus roles, cargan con el peso de las expectativas de sus padres y de la sociedad en general, y esto es una influencia importante en las decisiones finales.

De esta manera, es como la suma de diferentes factores determinan la decisión del estudiante hombre y mujer por continuar eligiendo especializaciones técnicas vincu- ladas a sus roles, o decidir por otras carreras. De igual forma, se reafirma que los roles de género aprendidos en la familia sí constituyen un elemento de influencia, pero no es el único factor que determina la elección.

\section{Conclusiones}

Existe una relación discordante entre lo que los estudiantes hacen y dicen sobre la igualdad de género; existe, es decir que no guardan armonía o similitud. Por una parte, lo que dicen revela cualidades de equidad, pues expresan que mujeres y hombres deben tener las mismas oportunidades; sin embargo, lo que hacen, o sus acciones, muestran que reproducen prácticas tradicionales de roles de género. De esta forma, se considera que existe una disonancia entre el "deber hacer" y el "deber ser".

Por lo tanto, se percibe un discurso emergente sobre igualdad de género, que está en un proceso de transición porque ha surgido a pesar de que los estudiantes han estado formados bajo los roles tradicionales en sus casas.

Esta es una de las principales conclusiones, ya que previamente no se consideró como un posible hallazgo; sin embargo, es importante destacarlo porque los jóvenes podrían estar frente a un proceso de cambio de postura sobre la equidad de género. A su vez, esto podría estar influenciado por las diferentes formaciones que se brindan en la escuela y en la comunidad, así como mensajes de medios masivos y sucesos sociales, como marchas por la igualdad de género.

En ese sentido, se recomienda implementar programas educativos enfocados en la igualdad de género dentro de las instituciones educativas, debido a que los y las estudiantes se encuentran en una etapa de definición de su identidad de género y estos estímulos en su formación podría complementar sus conocimientos.

Por otra parte, se identificaron casos disruptivos sobre la elección de especialización 
de Educación Media Técnica, específicamente aquellos que optaron por una especialización técnica que no estaba vinculada a sus roles de género. Se profundizó en su formación desde el hogar y se identificó que las mujeres que eligieron Industria e Innovación tuvieron un aprendizaje enfocado en la construcción y las matemáticas, orientado especialmente por su padre; mientras que los hombres que eligieron Bachillerato Técnico Vocacional en Salud crecieron más apegados a su mamá y desde su hogar realizaron funciones domésticas. De esta forma, ambos se salen del esquema tradicional de reproducción y aprendizaje de roles de género, por lo que permite ampliar las oportunidades de intervención en estos casos disruptivos donde hay una oportunidad de que el esquema de roles empiece a cambiar.

Se sugiere diseñar intervenciones educativas relacionadas con la igualdad de género con la participación de los padres y las madres de familia, pues ellos influyen en la formación $y$ en las decisiones de sus hijos e hijas.

Además, otra conclusión que abre una puerta para la intervención de la política educativa es que, luego de analizar los casos de los informantes, incluso los disruptivos, se identificó que, si bien los roles de género aprendidos en el hogar influyen en la elección de especialización técnica, no son determinantes. Es decir, que los estudiantes sí aprenden roles de género productivos de sus papás hombres, y reproductivos de sus mamás, y estos inciden en la elección de Educación Media Técnica. Sin embargo, existen otros factores externos que también influyen, tales como los modelajes y los aprendizajes previos a los cuales los y las estudiantes son expuestos desde pequeños.

Por lo tanto, sí existe una relación entre los roles de género y la elección de especialización técnica, pero es una relación vinculante, mas no determinante. Esto se refiere a que existe un vínculo muy estrecho entre los roles tradicionales con la elección de especialización técnica, pero no es la razón final para todos los casos.
Finalmente, resulta urgente formar a las niñas en la seguridad de sus capacidades y a los hombres en nuevas masculinidades. Las niñas necesitan un estímulo para estar seguras de que son capaces de estudiar especializaciones técnicas. Asimismo, los hombres pueden estar confiados en que pueden elegir cualquier especialización que deseen sin sentirse condicionados por los roles de género masculinos.

\section{Referencias bibliográficas}

Almagro Navarrete, B. P. (2017). Los roles de género en la familia y su incidencia en las relaciones interpersonales entre pares del estudiantado de segundo año de bachillerato del Colegio Nacional Amazonas de la ciudad de Quito [Tesis de Maestría]. Universidad de Central del Ecuador. http://www.dspace.uce. edu.ec/handle/25000/12580

Chaves Jiménez, A. R. (2012). Masculinidad y feminidad: ¿De qué estamos hablando? Revista Electrónica Educare, 16, 5-13. https://www.redalyc.org/ pdf/1941/194124704010.pdf

Comisión Económica para América Latina y Organización de las Naciones Unidas [CEPAL y ONU]. (2019). La Agenda 2030 y los Objetivos de Desarrollo Sostenible: una oportunidad para América Latina $y$ el Caribe. Objetivos, metas e indicadores mundiales. https://repositorio.cepal. org/bitstream/handle/11362/40155/24/ S1801141_es.pdf

Danermark, B., Jakobsen, L., Ekstrom, M. y Karlsson, J. (2016). Explicando la sociedad. El realismo crítico en las ciencias sociales. UCA Editores.

Dirección General de Estadística y Censos [DIGESTYC]. (2017). Encuesta de Hogares de Propósitos Múltiples. http://www.digestyc. gob.sv/index.php/temas/des/ehpm/publicaciones-ehpm.html

Fàbregues, S., Meneses, J., RodríguezGómez, D. y Paré, M.-H. (2016). Técnicas 
de investigación social y educativa. Editorial UOC.

Fondo de las Naciones Unidas para la Infancia [UNICEF]. (2018). Plan de Acción para la Igualdad entre los Géneros para 2018-2021. https://www.unicef.org/es/igualdad-de-genero/plan-de-accion-para-igualdadentre-los-generos-2018-2021

Galet Macedo, C. y Alzás García, T. (2014). Trascendencia del rol de género en la educación familiar. Campo Abierto, 33(2), 97-114. https://dialnet.unirioja.es/ servlet/articulo?codigo $=5250171$

Jansen, H. (2013). La lógica de la investigación por encuesta cualitativa y su posición en el campo de los métodos de investigación social. Paradigmas, 5(1), 39-72. https://dialnet.unirioja.es/servlet/ articulo?codigo $=4531575$

Martín-Baró, I. (1980). La imagen de la mujer en El Salvador. ECA, 35(380), 557-568. https://www.uca.edu.sv/coleccion-digital-IMB/wp-content/uploads/2015/11/1980La-imagen-de-la-mujer-en-El-SalvadorECA1980-35-380C.pdf
Ministerio de Educación [MINED]. (2020). Política de Equidad e Igualdad de Género. https://www.mined.gob.sv/descarga/politicaGenero/POLITICAS\%20DE\%20GENERO\%20 MINED\%20EDITADA.pdf

Parra, J. D. (2019). El arte del muestreo cualitativo y su importancia para la evaluación y la investigación de políticas públicas: una aproximación realista. OPERA, 25, 119-136. https://revistas.uexternado.edu.co/ index.php/opera/article/view/6020

Rocha Sánchez, T. E. (2009). Desarrollo de la Identidad de Género desde una Perspectiva Psico-Socio-Cultural: Un Recorrido Conceptual. Interamerican Journal of Psychology, 43(2), 250-259. https:// www.redalyc.org/pdf/284/28412891006.pdf

Rodrigues Bresque, M. L., Hoffmann Moreira, C., Mackedanz Flores, P. R. y Hoffmann Moreira, V. (2011). Cómo investigar cualitativamente. Entrevista y cuestionario. Contribuciones a las Ciencias Sociales, 11. https://www.eumed.net/rev/cccss/11/bmfm. htm 